SCIDic

\section{Oral Squamous Cell Carcinoma (OSCC) Associated With Various Forms Of Tobacco Usage}

Research Article

Meghana Reddy J ${ }^{1}$, Deepika Rajendran ${ }^{2 *}$

${ }^{1}$ Saveetha Dental College and Hospitals, Saveetha Institute of Medical and Technical Sciences (SIMATS), Saveetha University, Chennai, India.

${ }^{2}$ Senior Lecturer, Department of Oral Medicine and Radiology, Saveetha Dental College and Hospitals, Saveetha Institute of Medical and Technical Sciences (SIMATS), Saveetha University, Chennai, India.

\title{
Abstract
}

Oral squamous cell carcinoma (OSCC) is a pathological type of an epithelial lesion and is the most common neoplasm of oral cavity accounting for over $90 \%$ of oral cancers. The mortality from oral cancer remains high. From relative risk factors of alcohol and tobacco, it has been estimated that $75 \%$ of all oral cancers are preventable. In the remaining $25 \%$ of patients who are not exposed to these substances, the cause of their tumors remains unknown. The International Agency for Research on Cancer (IARC) confirmed that smoking of various forms of tobacco (e.g., bidis, pipes, cigars and cigarettes) is carcinogenic in humans. The aim of the study was to assess oral Squamous cell carcinoma (OSCC) associated with various forms of tobacco usage. A University-based study was conducted among 49 patients in the age group $32-71$. Data collection was done by reviewing the patient records and analysing the data of 49 patients from June 2019 to March 2020, excel tabulation and software analysis using spss was done. ,The data was then entered into Excel, tabulation was done and analyzed using SPSS and Pearson correlation was performed. There were Total 49 patients out of which 11 were female and 38 were male. The mean age was 53.2 years and age ranged from 32 to 71 years. Prevalence of various forms of tobacco were - $45.58 \%$ patients chewing pan, $17.64 \%$ smoking and $36.76 \%$ patients chewing gutka were affected with OSCC. Within the limits of this Study, it was concluded that Oral Squamous Cell Carcinoma (OSCC) was more prevalent in patients with pan chewing when compared with gutka and smoking.

Keywords: Oral Squamous Cell Carcinoma; Oral Cancer; Smokeless Tobacco; Smoking; Tobacco.

\section{Introduction}

Oral squamous cell carcinoma (OSCC) is a pathological type of an epithelial lesion and is the most common neoplasm of oral cavity accounting for over $90 \%$ of oral cancers [13]. It is the sequel of cellular changes, starting from cellular atypia which is the alteration in the cell epithelium, followed by dysplasia, leading to multiple cell involvement, causing carcinoma in situ and finally cell invasion and metastasis [36]. Cancer affects various parts of the oral cavity including lips, tongue, upper or lower gingiva, buccal mucosa, floor of mouth, hard palate, vestibule of mouth, retromolar trigone or major salivary glands [11]. Retained, unerupted third molar teeth have been associated with various pathological conditions. like cystic lesions, neoplasms, pericoronitis, periodontitis, dental caries, root resorption, and periapical infections [29].
The oral cavity is that part of the body which is easily accessible for direct visual examination but still the mortality from oral cancer remains high because in early stages the patient has no symptoms thus it could be used in early detection of precancerous and cancerous lesions. But either due to ignorance or inaccessibility of medical care, the disease gets detected in the later stages [19]. Therefore, the treatment for early detection of oral carcinomas is important, during the initial stages the treatment is more effective and the morbidity is minimal. One of the recent advances in the detection of premalignant lesions are by utilizing in-vivo toluidine blue staining with oral brush biopsy, thus enabling the clinician to choose the patients for scalpel biopsy [20].

Oral squamous cell carcinoma (OSCC) shows a complex, multi-

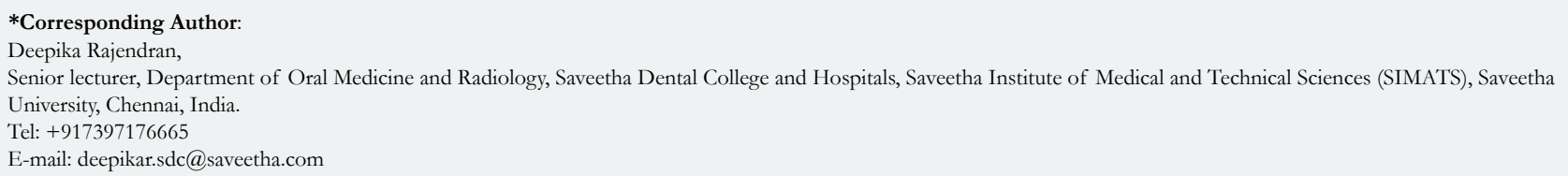

Copyright: Deepika Rajendran ${ }^{\circ} 2020$. This is an open-access article distributed under the terms of the Creative Commons Attribution License, which permits unrestricted use, distribution and reproduction in any medium, provided the original author and source are credited. 
stage developmental process, and involves several environmental and genetic factors [1, 9]. Among these factors, the important role of tobacco chewing, smoking and alcohol in oral malignancy has drawn increasing interest $[15,26]$.

Most of the invasive oral carcinomas are preceded by a premalignant stage that may last for many years. Classification of tumor progression is normal, hyperplastic, dysplastic, carcinoma in situ and invasive carcinoma. The majority of the initial alterations of precancerous and cancerous oral lesions are not readily recognizable, on clinical or histopathological examination. The basic biology of initiation and progression of these tumors is still obscure $[22,6]$.

The risk factors included tobacco associated intra-oral carcinogens, which may play a synergistic role in oral tumorigenesis. The main risk factors are alcohol and tobacco, it has been estimated that over $75 \%$ of all oral cancers are preventable. In the remaining $25 \%$ mostly the patients who are not exposed to these substances, in that case the cause of their tumors remains unknown [35]. The disproportionately higher incidence of carcinoma of the headneck in reference to other malignancies in India, could be due to use of tobacco in various forms, consumption of alcohol, low socioeconomic condition related to poor hygiene, poor diet and rampant viral infections [8].

Oral neoplasia has been related to chewing tobacco with betel quid in India and other asian countries, whereas in western countries, cigarette smoking and heavy alcohol consumption are the main risk factors [18]. The international agency for research on cancer (IARC) confirmed that smoking of various forms of tobacco (e.g., bidis, pipes, cigars and cigarettes) is carcinogenic in humans [11]. Smokeless tobacco has increased risk of oral cancer and potentially malignant disorders [37]. Chewing of tobacco with betel quid increases exposure to carcinogenic tobacco-specific nitrosamines (TSNA) and to nitrosamines derived from areca nut alkaloids. Furthermore, reactive oxygen species (ROS) implicated in multistage carcinogenesis, are also generated in substantial amounts in the oral cavity during chewing. Tobacco smoke pro-carcinogens like benzo-[a]-pyrene, are metabolized by oxidizing enzymes, particularly cytochrome p450, some leading to the production of reactive carcinogenic intermediates. Some studies link that cytochrome P450 family 1, subfamily A (CYP1A1) and CYP2E1 genotype, shows susceptibility to oral cancer [14].

Alcohol, acting both independently and also synergistically with smoking, has been implicated in oral carcinogenesis. The important aspect of alcohol is that it may act as a solvent and improve the penetration of carcinogens into target tissues. Acetaldehyde, which is the alcohol metabolite, has been identified recently as a tumor promoter [3].

Good oral hygiene has to be maintained and the vast majority of the students were not smoking, creating awareness among the students is very important for the overall health of the individual [32]. Advanced training is important to treat various diseases in Oral medicine [31].

Medication-related osteonecrosis of the jaw (MRONJ) is increasing in frequency among patients who are on bisphosphonate therapy, management of such complications is very important [24]. Dental practitioners should be aware of the extra root in mandibular first molar which is rare [27]. Trigeminal neuralgia is characterized by unilateral episodic and lancinating pain, tumors can be present in the brain stem.MRI is the gold standard imaging for identifying these lesions [33].

The importance of this research was to find out the risk factors of various forms of tobacco with oral squamous cell carcinoma, and to detect the lesion in early stages. The aim of the study was to assess oral Squamous cell carcinoma (OSCC) associated with various forms of tobacco usage.

\section{Materials and Methods}

A University-based study was conducted among 49 patients in the age group of $32-71$ years where two people are involved (1 guide and 1 Student). Data retrieval was easier because of similar ethnicity and specific time period. The disadvantage was that the patients may not report back for a review.

Bias was avoided by including all the data available. The Confounding factors were eliminated and the results can be applied to practical situations.

The study was conducted with the approval of the Institutional Ethics Committee [SDC/SIHEC/2020/DIASDATA/0619-0320]. Data collection was done by reviewing the patient records and analysing the data of 49 patients from June 2019 to March 2020, excel tabulation, statistical analysis using spss and Pearson correlation was performed in oral squamous cell carcinoma patients with smoking tobacco and smokeless tobacco. Statistical analysis was done by exporting the data to SPSS for data checking. Data was sorted and then represented in frequencies. Calculating frequencies and sorting. Descriptive results were presented using mean, tables and graphs.

Inclusion criteria includes patients with histopathologically proven oral squamous cell carcinoma (OSCC) patients and the exclusion criteria includes other malignant tumors.

\section{Results And Discussion}

There were 49 cases of oral squamous cell carcinoma during the 10 months of study; out of these, $38(77.5 \%)$ were male and $11(22.45 \%)$ were female. According to age, the highest incidence was in the age group of fourth to sixth decades of life. The mean age is 53.2 years and age ranged from 32 to 71 years. According to the prevalence of habit, the majority of patients who were having a habit of tobacco chewing were found to develop OSCC. (Fig. 1) describes the distribution of OSCC among the study population by age. It was observed that the highest was in the age group of 41-50 years, and this relationship was found to be statistically significant $(\mathrm{P} \leq 0.05)$. Similarly, on gender-wise comparison, 38 males exhibited Oral cancer explains the relationship of adverse habits and gender. Males had the highest prevalence of gutkha chewing, and similar findings were observed in females (Fig. 2).

Prevalence of various forms of tobacco were - $45.59 \%$ patients chewing pan, $17.65 \%$ smoking and $36.76 \%$ patients chewing gutka were affected with OSCC (Fig 3).

The incidence of oral cancer varies according to the different 
Figure 1. Bar graph showing the descriptive distribution of Oral squamous cell carcinoma among the participants with age. Highest prevalence of age groups was seen in $41-50$ years with $38.78 \%$. The $X$ axis represents the age of the participants and the $Y$ axis represents the number of participants in each age group.

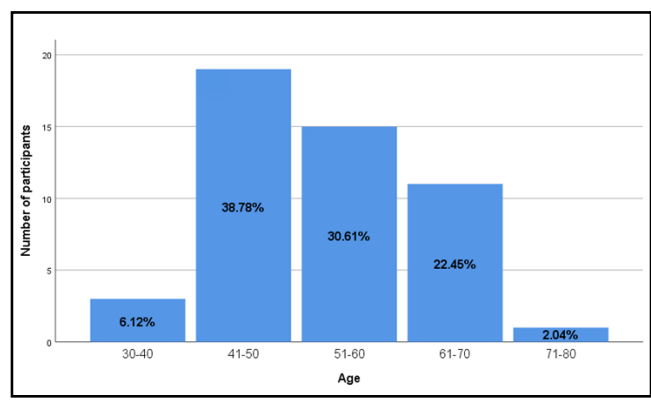

Figure 2. Bar graph showing the descriptive distribution of Oral squamous cell carcinoma among the participants with gender where red colour represents male with $77.55 \%$ and blue colour represents female with $22.45 \%$. The predominant gender is male. The $\mathrm{X}$ axis represents the gender distribution of participants and the $\mathrm{Y}$ axis represents the number of participants.

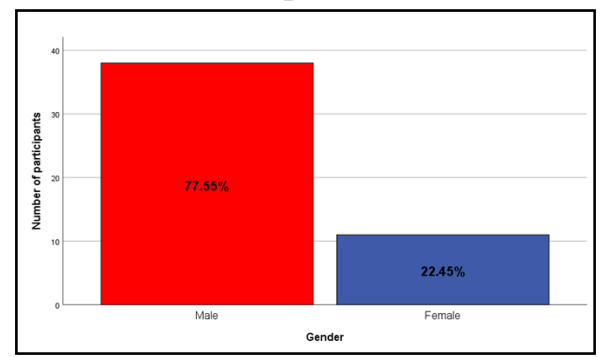

Figure 3. This graph shows the association between various forms of tobacco usage and Oral Squamous cell carcinoma, where red colour represents pan chewing with $45.59 \%$, blue colour represents smoking with $17.65 \%$ and green colour represents gutka with $36.76 \%$. The prevalent habit was pan chewing. The $\mathrm{X}$ axis represents the Oral squamous cell carcinoma (OSCC) patients and the $\mathrm{Y}$ axis represents the frequency of various forms of tobacco usage like pan chewing, smoking and gutka. Pearson correlation was done $P=0.020$ for pan chewing $(<0.05$ - indicating statistically significant $), P=0.308$ for smoking ( $>0.05$ - indicating statistically not significant) and $P=0.648$ for gutka $(>0.05$ - indicating statistically not significant).

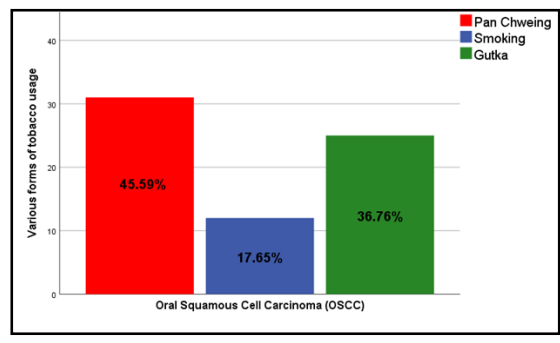

geographical locations, this could be due to the differences in lifestyles, culture and developmental status. Metastatic malignancies are rare lesions of the oral cavity. The primary lesion can metastasize into various organs like lungs, liver, breast, kidney [23].

In the present study, out of 49 OSCC cases, $77.5 \%$ were males and $22.4 \%$ were females, with the largest number of OSCC developing in the fourth and fifth decades of life. This is in accordance to a study conducted by Sharma et al. in Uttar Pradesh, in which $68.7 \%$ of males had OSCC, which is less as compared to females $(31.2 \%)$ [30].

Mehrotra et al. confirmed that OSCC in North India is a disease of middle-aged men, which may be due to changing social habits in high socioeconomic groups or cultural habits of some rural areas of India [21, 12]. Furthermore, in the present study, 2 patients belonged to the age group of $30-40$ years, whereas in other studies, the majority of cases were aged $>50$ years. This shows the alarming factor regarding tobacco consumption and OSCC cases in relation to age, which is dropping at a significant rate as mentioned in the present study.

Tobacco is an independent risk factor, the relative risk of occurrence of OSCC in tobacco users is 11 times that of people who had never used tobacco. Tobacco is used in smoking as well as smokeless forms. Smoking bidi is an important risk factor for OSCC. Bidi smokers have 3.1 times increased risk for oral cancer compared to nonsmokers in Southeast Asia.

In the present study $45.31 \%$ patients who were chewing pan, $37.50 \%$ patients who were chewing gutka, whereas only $17.19 \%$ smokers were affected with OSCC. OSCC is largely associated with gutka followed by tobacco flakes, gutka, and pan masala which are termed poly ingredient oral dip products and are more carcinogenic because of other carcinogenic products present. This could be due to the fact that tobacco and other carcinogenic compounds are kept in contact with oral mucosa for a considerably longer time [16].

In this study, those who chewed tobacco were also found to 
smoke heavily, which further exaggerated the effect and the risk of developing oral cancer [28]. Tobacco chewing is a stronger risk factor for oral cancer than smoking where chewing is a prevalent practice. The clinicopathologic profile of Indian oral cancers shows significant differences from oral cancer in several developed countries of the world, including the USA, the UK, France, and Japan where it is associated with tobacco smoking with or without alcohol consumption [2, 24].

Matrix metalloproteinase-9 (MMP-9) is an enzyme that is considered as the early tissue changes that happen due to various habits such as smoking tobacco, chewing tobacco or stress in potentially malignant disorders (OPMDs). These changes in the tissues alter the expression of MMP-9.The expression of MMP-9 has proved to be a diagnostic marker in oral cancer in the tissue, serum and saliva $[7,34]$. The upregulated salivary miRNA 184, and miRNA 21 and downregulated salivary miRNA 145 can be used as potential biomarkers to predict malignancy. These can be used in early diagnosis [17].

One of the main complications of non surgical treatment of oral cancer is oral mucositis. It is considered an inherent outcome of chemotherapy or radiotherapy to the head and neck region in cancer patients. Topical application of Vitamin E creams showed better results than systemic intake of vitamin E [4]. Post operative pain is common after surgical intervention, even after intake of analgesics pain may not subside. Vitamin $C$ has shown to exhibit promising analgesic effects [5].

\section{Limitations}

The limitations of this study were alcohol consumption and other tobacco product usage was not included,sample size was less, specific population was covered and other diseases were not evaluated.

\section{Future scope}

Education about the harmful effects of smokeless tobacco and smoke tobacco usage, other parts of the population should be covered.

\section{Conclusion}

Within the limits of this Study, It was concluded that Oral Squamous Cell Carcinoma (OSCC) was more prevalent in patients with pan chewing when compared with gutka and smoking. The prevalence of OSCC showed an alarming rise among the younger population; hence, it is important to undertake programs to prevent and control OSCC by screening for early diagnosis and by promoting a tobacco-free environment. It is also very important to improve the living standards of people where access to health care is poor or limited. Race, ethnicity, and age cannot be altered; however, lifestyle behavior and habits such as tobacco usage, smoking, and alcohol are amenable to change, all of which could be initiated through health education.

\section{References}

[1]. Bagan J, Sarrion G, Jimenez Y. Oral cancer: clinical features. Oral Oncol. 2010 Jun;46(6):414-7. Pubmed PMID: 20400366

[2]. Balaram P, Sridhar H, Rajkumar T, Vaccarella S, Herrero R, Nandakumar
A, et al. Oral cancer in southern India: the influence of smoking, drinking, paan-chewing and oral hygiene. Int J Cancer. 2002 Mar 20;98(3):440-5. Pubmed PMID: 11920597.

[3]. Blot WJ. Alcohol and cancer. Cancer Res. 1992 Apr 1;52(7 Suppl):2119s2123s. Pubmed PMID: 1544150.

[4]. Chaitanya NC, Muthukrishnan A, Babu DBG, Kumari CS, Lakshmi MA, Palat G, et al. Role of Vitamin E and Vitamin A in Oral Mucositis Induced by Cancer Chemo/Radiotherapy- A Meta-analysis. J Clin Diagn Res. 2017 May;11(5):ZE06-ZE09. Pubmed PMID: 28658926.

[5]. Chaitanya NC, Muthukrishnan A, Krishnaprasad CMS, Sanjuprasanna G, Pillay P, Mounika B. An Insight and Update on the Analgesic Properties of Vitamin C. J Pharm Bioallied Sci. 2018 Jul-Sep;10(3):119-125. Pubmed PMID: 30237682.

[6]. Choudhury P, Panigrahi RG, Maragathavalli, Panigrahi A, Patra PC. Vanishing roots: first case report of idiopathic multiple cervico-apical external root resorption. J Clin Diagn Res. 2015 Mar;9(3):ZD17-9. Pubmed PMID: 25954713.

[7]. Dharman S, Muthukrishnan A. Oral mucous membrane pemphigoid - Two case reports with varied clinical presentation. J Indian Soc Periodontol. 2016 Nov-Dec;20(6):630-634. Pubmed PMID: 29238145.

[8]. Franceschi S, Bidoli E, Herrero R, Muñoz N. Comparison of cancers of the oral cavity and pharynx worldwide: etiological clues. Oral Oncol. 2000 Jan;36(1):106-15. Pubmed PMID: 10889929.

[9]. Hillbertz NS, Hirsch JM, Jalouli J, Jalouli MM, Sand L. Viral and molecular aspects of oral cancer. Anticancer Res. 2012 Oct;32(10):4201-12. Pubmed PMID: 23060540.

[10]. IARC Working Group on the Evaluation of Carcinogenic Risks to Humans. Betel-quid and areca-nut chewing and some areca-nut derived nitrosamines. IARC Monogr Eval Carcinog Risks Hum. 2004;85:1-334. Pubmed PMID: 15635762.

[11]. IARC Working Group on the Evaluation of Carcinogenic Risks to Humans, World Health Organization, International Agency for Research on Cancer. Tobacco smoke and involuntary smoking. Iarc; 2004.

[12]. Jayalekshmi PA, Gangadharan P, Akiba S, Koriyama C, Nair RR. Oral cavity cancer risk in relation to tobacco chewing and bidi smoking among men in Karunagappally, Kerala, India: Karunagappally cohort study. Cancer Sci. 2011 Feb;102(2):460-7. Pubmed PMID: 21129124.

[13]. Johnson NW, Jayasekara P, Amarasinghe AA. Squamous cell carcinoma and precursor lesions of the oral cavity: epidemiology and aetiology. Periodontol 2000. 2011 Oct;57(1):19-37. Pubmed PMID: 21781177.

[14]. Kawajiri K, Fujii-Kuriyama Y. P450 and human cancer. Jpn J Cancer Res. 1991 Dec;82(12):1325-35. Pubmed PMID: 1778754.

[15]. Lissowska J, Pilarska A, Pilarski P, Samolczyk-Wanyura D, Piekarczyk J, Bardin-Mikoltajczak A, et al. Smoking, alcohol, diet, dentition and sexual practices in the epidemiology of oral cancer in Poland. Eur J Cancer Prev. 2003 Feb;12(1):25-33. Pubmed PMID: 12548107.

[16]. Madani AH, Dikshit M, Bhaduri D. Risk for oral cancer associated to smoking, smokeless and oral dip products. Indian J Public Health. 2012 JanMar;56(1):57-60. Pubmed PMID: 22684175.

[17]. Maheswari TNU, Venugopal A, Sureshbabu NM, Ramani P. Salivary micro RNA as a potential biomarker in oral potentially malignant disorders: A systematic review. Ci Ji Yi Xue Za Zhi. 2018 Apr-Jun;30(2):55-60. Pubmed PMID: 29875583.

[18]. Manjari M, Popli R, Paul S, Gupta VP, Kaholon SK. Prevalence of oral cavity, pharynx, larynx and nasal cavity malignancies in Amritsar, Punjab. Indian Journal of Otolaryngology and Head and Neck Surgery. $1996 \mathrm{Jul}$ 1;48(3):191-5.

[19]. McCullough MJ, Prasad G, Farah CS. Oral mucosal malignancy and potentially malignant lesions: an update on the epidemiology, risk factors, diagnosis and management. Aust Dent J. 2010 Jun;55 Suppl 1:61-5. Pubmed PMID: 20553246.

[20]. Mehrotra R, Gupta A, Singh M, Ibrahim R. Application of cytology and molecular biology in diagnosing premalignant or malignant oral lesions. Mol Cancer. 2006 Mar 23;5:11. Pubmed PMID: 16556320.

[21]. Mehrotra R, Singh MK, Pandya S, Singh M. The use of an oral brush biopsy without computer-assisted analysis in the evaluation of oral lesions: a study of 94 patients. Oral Surg Oral Med Oral Pathol Oral Radiol Endod. 2008 Aug;106(2):246-53. Pubmed PMID: 18644521.

[22]. Meyskens Jr FL. Biology and intervention of the premalignant process. Cancer Bull. 1991;43:475-80.

[23]. Misra SR, Shankar YU, Rastogi V, Maragathavalli G. Metastatic hepatocellular carcinoma in the maxilla and mandible, an extremely rare presentation. Contemp Clin Dent. 2015 Mar;6(Suppl 1):S117-21. Pubmed PMID: 25821363.

[24]. Muthukrishnan A, Bijai Kumar L. Actinic cheilosis: early intervention prevents malignant transformation. BMJ Case Rep. 2017 Mar 20;2017:bcr2016218654. Pubmed PMID: 28320702. 
[25]. Muthukrishnan A, Bijai Kumar L, Ramalingam G. Medication-related osteonecrosis of the jaw: a dentist's nightmare. BMJ Case Rep. 2016 Apr 6;2016:bcr2016214626. Pubmed PMID: 27053542.

[26]. Muwonge R, Ramadas K, Sankila R, Thara S, Thomas G, Vinoda J, et al. Role of tobacco smoking, chewing and alcohol drinking in the risk of oral cancer in Trivandrum, India: a nested case-control design using incident cancer cases. Oral Oncol. 2008 May;44(5):446-54. Pubmed PMID: 17933578.

[27]. Patil SR, Maragathavalli G, Araki K, Al-Zoubi IA, Sghaireen MG, Gudipaneni RK, et al. Three-rooted mandibular first molars in a Saudi Arabian population: A CBCT study. Pesquisa brasileira em odontopediatria e clinica integrada. 2018 Aug 27;18(1):4133.

[28]. Rodu B, Jansson C. Smokeless tobacco and oral cancer: a review of the risks and determinants. Crit Rev Oral Biol Med. 2004 Sep 1;15(5):252-63. Pubmed PMID: 15470264.

[29]. Rohini S, Kumar VJ. Incidence of dental caries and pericoronitis associated with impacted mandibular third molar-A radiographic study. Research Journal of Pharmacy and Technology. 2017 Apr 1;10(4):1081.

[30]. Sharma P, Saxena S, Aggarwal P. Trends in the epidemiology of oral squamous cell carcinoma in Western UP: an institutional study. Indian J Dent Res. 2010 Jul-Sep;21(3):316-9. Pubmed PMID: 20930335.

[31]. Steele JC, Clark HJ, Hong CH, Jurge S, Muthukrishnan A, Kerr AR, et al. World Workshop on Oral Medicine VI: an international validation study of clinical competencies for advanced training in oral medicine. Oral Surg Oral Med Oral Pathol Oral Radiol. 2015 Aug;120(2):143-51.e7. Pubmed PMID: 25861956.

[32]. Subashri A, Maheshwari TU. Knowledge and attitude of oral hygiene practice among dental students. Research Journal of Pharmacy and Technology. 2016 Nov 1;9(11):1840.

[33]. Subha M, Arvind M. Role of magnetic resonance imaging in evaluation of trigeminal neuralgia with its anatomical correlation. Biomedical and Pharmacology Journal. 2019 Mar 25;12(1):289-96.

[34]. Venugopal A, Uma Maheswari TN. Expression of matrix metalloproteinase- 9 in oral potentially malignant disorders: A systematic review. J Oral Maxillofac Pathol. 2016 Sep-Dec;20(3):474-479. Pubmed PMID: 27721614.

[35]. Walker DM, Boey G, McDonald LA. The pathology of oral cancer. Pathology. 2003 Oct;35(5):376-83. Pubmed PMID: 14555380.

[36]. Warnakulasuriya S, Johnson NW, van der Waal I. Nomenclature and classification of potentially malignant disorders of the oral mucosa. J Oral Pathol Med. 2007 Nov;36(10):575-80. Pubmed PMID: 17944749.

[37]. Muthukrishnan A, Warnakulasuriya S. Oral health consequences of smokeless tobacco use. Indian J Med Res. 2018 Jul;148(1):35-40. Pubmed PMID: 30264752 . 